


\title{
New Tips and Tricks in Stapled Hemorrhoidopexy for Management of Symptomatic Hemorrhoids
}

\author{
Abd-Allah H. Ibrahim, Mohab G Elbarbary and Mahmoud Abdelbaky Mahmoud \\ Department of General Surgery, Faculty of Medicine, Ain shams University, Egypt
}

\begin{abstract}
Background: Hemorrhoids is a common anorectal disease defined as the symptomatic enlargement and/or distal displacement of anal cushions. A surgical option would be considered in symptomatic patients with grade III \& grade IV hemorrhoids and complicated grade II. The conventional surgical techniques such as Milligan- Morgan's open hemorrhoidectomy and Ferguson's closed hemorrhoidectomy were preferred choice for the treatment of these patients till the evolvement of stapled hemorrhoidopexy $(\mathrm{SH})$ by Longo in 1998. In this study we presented our experience in managing these patients using the $\mathrm{SH}$ technique aiming to offer (Tips and Tricks) to improve the outcome of this technique and to evaluate its effectiveness in long term as a new procedure for patients with hemorrhoids.
\end{abstract}

Patients and methods: We operated upon 300 patients with symptomatic hemorrhoids who presented from June 2013 to June 2016. The procedure was done in Ain-Shams University hospitals and Dar el Hekma hospital. The gun used in all cases was the same (PPH 03, Ethicon Endo Surgery, Cincinnati, Ohio).

All patients were followed up after operation for 2 years.

Results: Our results were up to the expectations as shown by absence of major complications and minimal postoperative pain and bleeding. This outcome was due to standardization of the technique regardless of the surgeon, and addition of some tricks.

Conclusions: Stapled hemorrhoidopexy $(\mathrm{SH})$ is a safe procedure, which takes less operative time, less hospital stay, and with no major complications provided that the technique is conducted with vigilance and diligence.

Keywords: Hemorrhoids, Stapled hemorrhoidopexy, Open hemorrhoidectomy.

\section{Introduction}

Hemorrhoids is a common anorectal disease defined as the symptomatic enlargement and/or distal displacement of anal cushions, ${ }^{1}$ It is clinically characterized by painless rectal bleeding during defecation with or without prolapsing anal tissue. ${ }^{2}$

Internal hemorrhoids develop when cushions of vascular tissue in the anus undergo pathological change. These cushions have an important role in maintaining continence because they function along with the internal anal sphincter to allow the complete closure of the anal canal. It is thought that around $70 \%$ of adult population above 30 years of age is affected with this disease. It is more common in males with maximal incidence between 45 and 65 years of age. ${ }^{3}$

Classically there are three main cushions located at left lateral, right posterior and right anterior positions. ${ }^{3}$ Hemorrhoids are generally classified by their location; internal (originates above the dentate line and covered by anal mucosa), external (originates below the dentate line and covered by anoderm) and mixed type. ${ }^{4}$ Internal hemorrhoids are further graded based on their appearance and degree of prolapse. ${ }^{5}$

Treatment options mainly depend on the type and severity of hemorrhoids, patient's preference and the experience of physicians. External hemorrhoid usually requires no specific treatment unless it becomes acutely thrombosed or causes patients discomfort. $^{6}$

Low-graded internal hemorrhoids are effectively treated with dietary and lifestyle modification, conservative treatment of hemorrhoids and some preventive measures. ${ }^{7}$

The advice usually includes increasing the intake of dietary fiber and oral fluids, having regular exercise, avoid straining on the toilet, and avoiding drug causing constipation or diarrhea. ${ }^{1}$

The main goal of medical treatment is to control acute symptoms of hemorrhoids rather than to cure the underlying hemorrhoids. There are several modern drugs including pills, suppositories, cream and wipes. ${ }^{8}$ Meanwhile, low-grade internal hemorrhoids 
can be effectively treated with medication and nonoperative measures (such as rubber band ligation and injection sclerotherapy). ${ }^{9}$

Many office-based procedures (such as rubber band ligation, injection sclerotherapy, infrared coagulation, cryotherapy, radiofrequency ablation and laser therapy) are effectively performed for grade I- II hemorrhoids and some cases of grade III hemorrhoids. ${ }^{10}$ Surgical intervention is usually required in low-graded hemorrhoids refractory to non-surgical treatment, high-graded symptomatic hemorrhoids, and hemorrhoids with complication such as strangulation and thrombosis. ${ }^{11}$

Two well-established methods of hemorrhoidectomy are widely used, the open (Milligan-Morgan) ${ }^{12}$ and closed (Ferguson) ${ }^{13}$ techniques are especially popular. ${ }^{14}$ Both procedures involve an anodermal wound and are associated with significant pain, marked bleeding, and a prolonged period before return to normal activity. ${ }^{15}$ In order to minimize or avoid post-hemorrhoidectomy pain, more recent approaches are used, including Ligasure hemorrhoidectomy, Doppler-guided hemorrhoidal artery ligation and stapled hemorrhoidopexy. ${ }^{16}$ Stapled hemorrhoidopexy is a technique used to excise redundant mucosal tissue and resuspend the hemorrhoids into the anal canal, using a specific circular stapling device. ${ }^{5}$ This technique has the potential advantages of reduction of operating time, postoperative pain, hospital stay and time to return to work. ${ }^{17,18}$

\section{Patients and methods}

We operated upon 300 patients with symptomatic hemorrhoids (Figures 1-3) who presented to outpatient surgical departments from June 2013 to June 2016.

The procedures were done in Ain-Shams University hospitals (El-Demerdash hospital, and Ain Shams university specialized hospital Cairo, Egypt) and Dar El Hekma hospital. The gun used in all cases was the same (PPH 03, Ethicon Endo Surgery, Cincinnati, Ohio).

All patients were followed up after operation for 2 years.

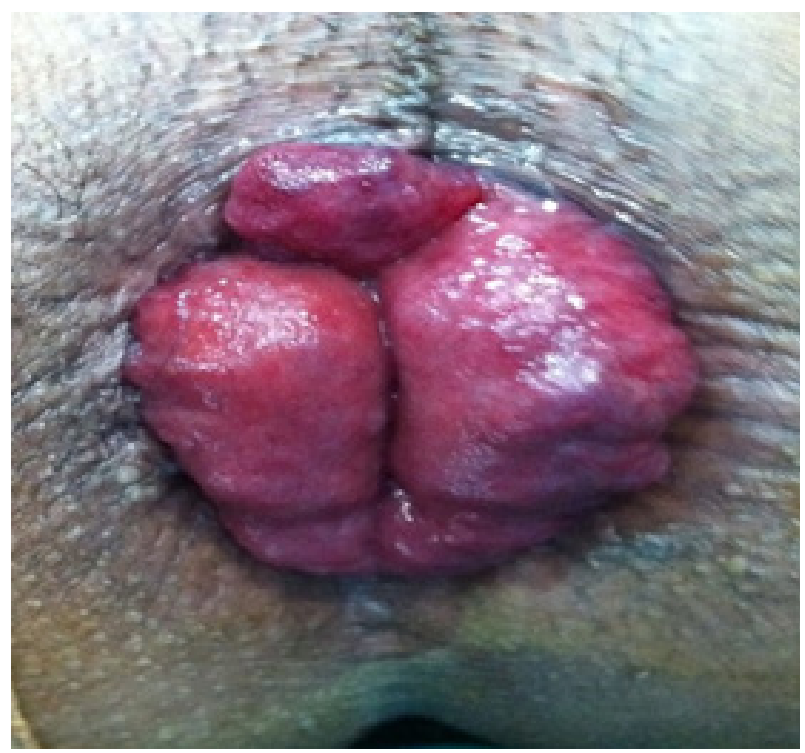

Fig 1.

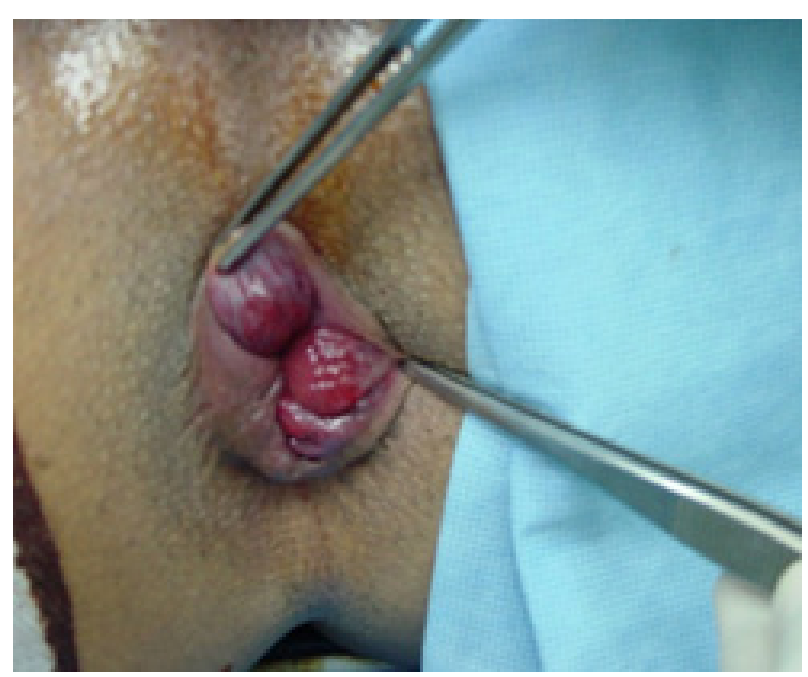

Fig 2.

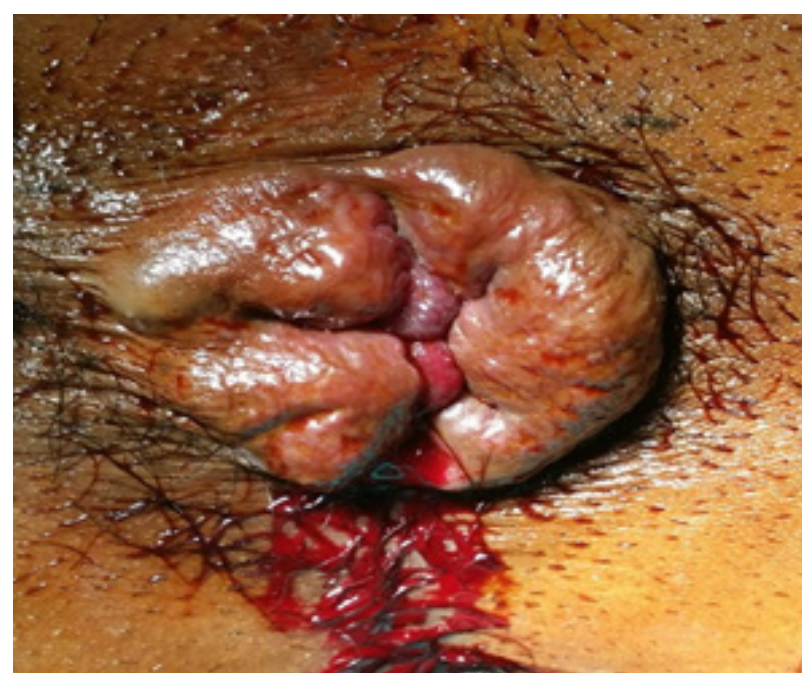

Fig 3. 


\section{Inclusion criteria}

Patients presented to us with symptomatic 3rd or 4th degree hemorrhoids, and complicated or refractory 2nd degree were included in the study. Also patients with recurrent hemorrhoids were included.

\section{Exclusion criteria}

Patients who had not completed all follow up visits were excluded. Patients with preoperative anal stricture, fecal incontinence, proctitis, rectal and peri-anal sepsis, anal fissure were also excluded.

Patients with rectal and anal varices proved by preoperative colonoscopy were excluded.

\section{Steps of the study \\ Preoperative evaluation}

Patients were classified according to the degree of haemorrhoids:

- 52 patients (17.3\%) had complicated grade II haemorrhoids.

- 161 patients $(53.7 \%)$ had grade III haemorrhoids.

- 87 patients (29\%) had grade IV haemorrhoids.

A detailed history of the presenting complaint, physical examination, routine investigations as well as anoscopy were carried out for all patients. Colonoscopy was ordered for patients above 40 years to exclude any colorectal pathologies.

Pain was assessed by visual analogue score (VAS).

\section{Operative steps}

- $\quad$ Routine pre-operative antibiotic was used (2nd generation cephalosporin)

- General anesthesia was used for 276 and spinal for 24 cases

- Lithotomy position

- Dicynone $250 \mathrm{mg}$ IV at the start of the procedure.

- Good strapping of the gluteal folds.

- Routine proctoscopy reassessment and lavage

- Fixation of our modified circular anal dilator with extra openings (Figures. $\mathbf{4 a} \mathbf{a} \mathbf{b}$ ).

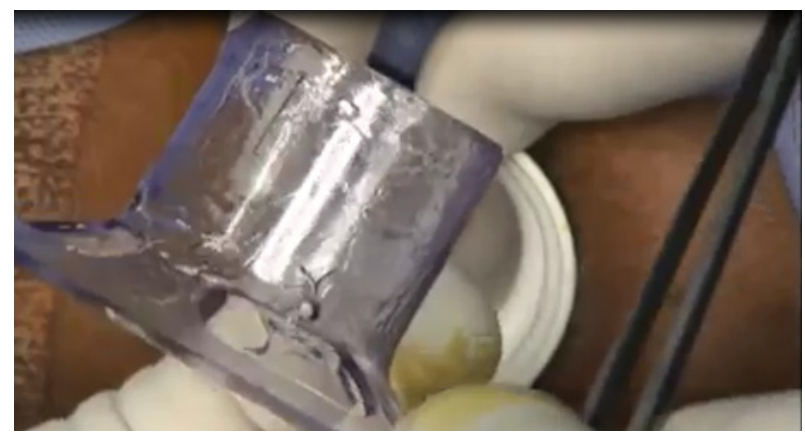

Fig 4a: Our modification on the anal dilator by extra-holes.

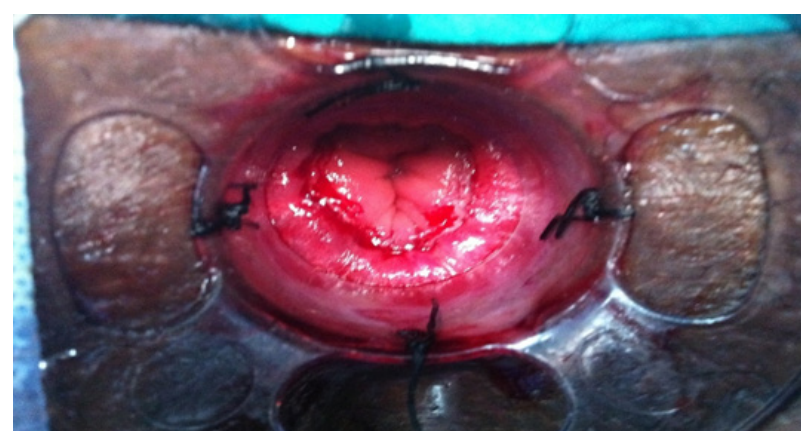

Fig 4b: Circular anal dilator with extra-opening (excellent fixation).

- Introduction of purse string suture anoscope (not used in case of huge prolapsed piles).

- Purse string at $1 \mathrm{~cm}$ above the edge of the circular anal dilator (corresponding to $1 \mathrm{~cm}$ above the dentate line) using proline $3 / 0$ with mainly sub-mucosal bites and without in situ rotation of the dilator to avoid any gaps (Figure 5).

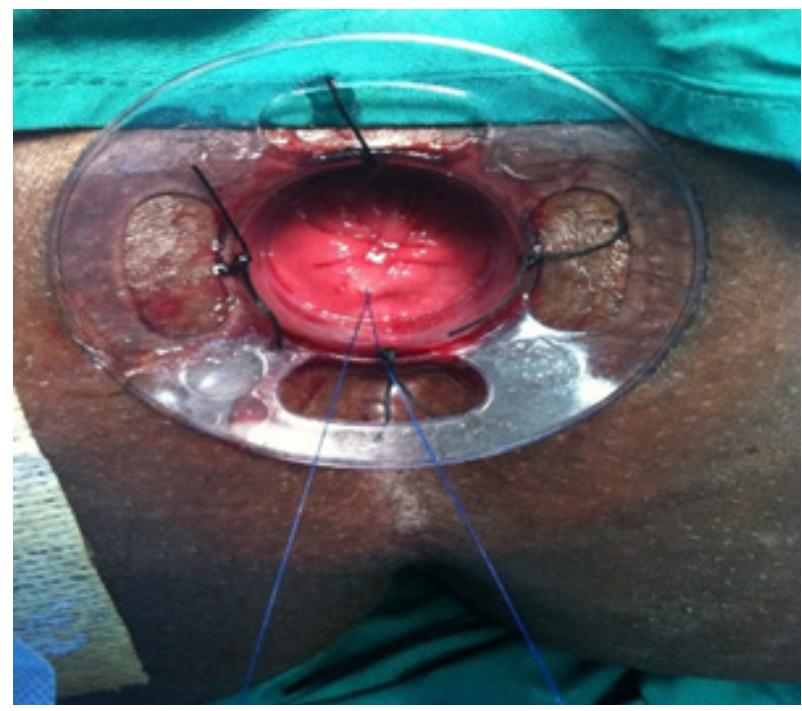

Fig 5: Completion of the purse-string using proline $3 / 0$.

- Gentle introduction of the fully opened gun. The anvil was lubricated and well introduced beyond the purse-string. Tightening of the purse-string around the shaft of the stapler. The gun was closed gently until detection of the indicator at the green zone of the gun. Measure of $4 \mathrm{~cm}$ shown on the stapler head indicates that our staple line will be high (Figure 6). 


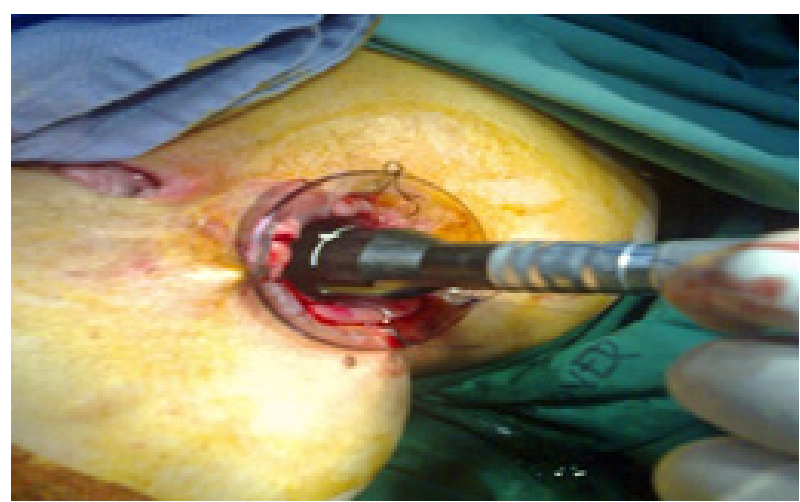

Fig 6: Gun length $4 \mathrm{~cm}$ indicates that your staple line will be high.

- After 20 seconds we fired the gun one shot with maintained power. The gun was gently extracted and checking the doughnut on the shaft is important before changing its orientation (Figure 7).

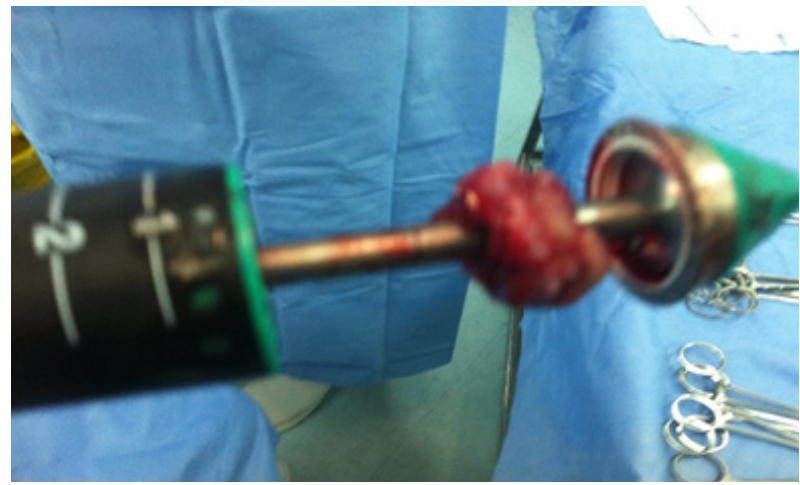

Fig 7: Complete circular doghnuts without changing gun orientation.

- We routinely re-introduced the purse string suture anoscope again to check the staple line. Additional hemostatic or reinforcement stitches using vicryl $3 / 0$ were used only if needed (Figure 8).

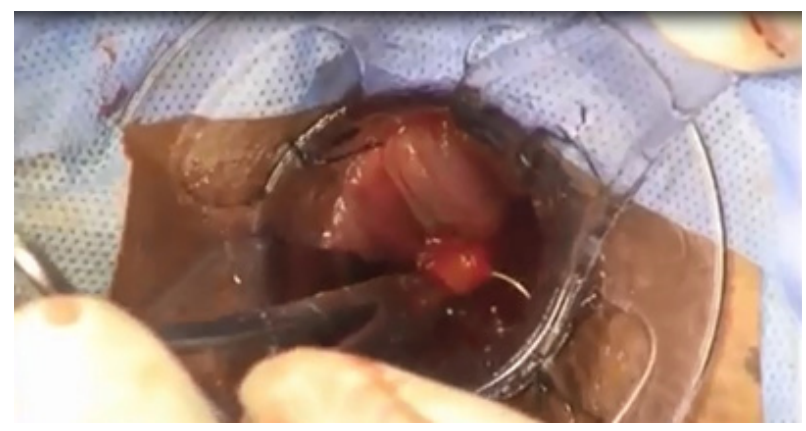

Fig 8: hemostatic reinforcement suture.
- Spongstan (Hemostatic sponges) was used as gentle pack and dicynone $250 \mathrm{mg}$ was injected through it. This pack was removed on next day morning with sitting in a warm bath before discharge (Figure 9).

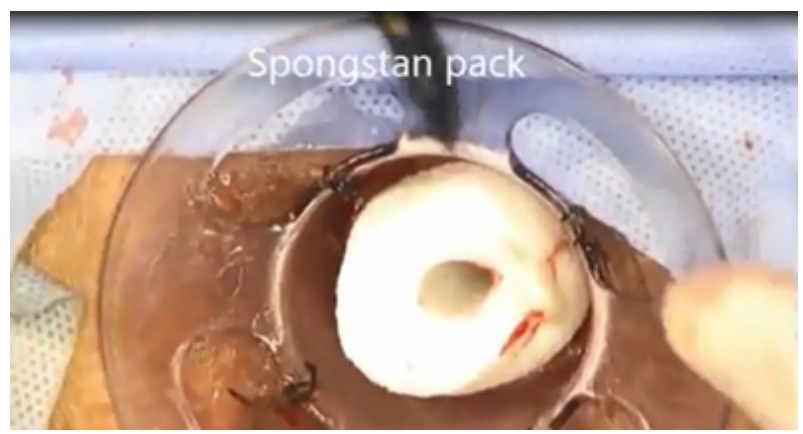

Fig 9: Spongstan pack.

- Our protocol was to give postoperative Daflon for 2-3 weeks to prevent bleeding, laxative for 2 weeks to prevent constipation and straining, and non-steroidal anti-inflammatory agent for few days for pain relief.

\section{Results}

We operated on 300 patients having symptomatic hemorrhoids using stapled technique.

The 300 cases distribution according to sex, type of surgery and degree of hemorrhoids is demonstrated in Table 1.

The mean age was 34 years (range 18 to 72 years) The mean operative time was 23 min (range from 15 minutes to $30 \mathrm{~min}$ ).

The results were as follows:

- Complete and ideal width----279 cases (93\%).

- Complete and small width ---- 6 cases (2\%).

- Incomplete ----15 cases (5\%).

- Smooth muscle in specimen ---30 cases (10).

- Hemostatic or reinforcement sutures were estimated to be necessary for 39cases (13\%).

- Hospital stay was one day for 282 patients $(94.1 \%)$ and 2 to 4 days for 18 patients $(5.9 \%)$.

- $\quad$ Follow up was for 2 years in 273. One hundred ten patients completed one year follow up. Thirty four patients only followed for 6 months. The mean follow up period was 20 months.

Complications are shown in Table 2.

We didn't have any major problems as stricture, stapler malfunction, sphincter injury, anastomotic dehiscence, jammed stapler, rectal perforation or 
septic complications.

In our study recurrence of prolapse occurred in 19 patients $(6.4 \%)$ but without symptoms. Fifteen patients $(4.9 \%)$ had recurrent prolapse with symptoms.

We re-operated 13 patients (4.3\%): 12 patients (4\%) for symptomatic prolapse after one year and 1 patient $(0.3 \%)$ for persistent brisk bleeding within first 24 hours who was treated by taking stitches over bleeding site.

Pain was measured by visual analogue scale (VAS) during hospital stay and follow up visits at 2 weeks, 1 month, 3 months, 6 months, 1 year and 2 years.

\section{Statistical analysis}

Data were collected on specific proforma and were analyzed by SPSS version 11. Qualitative variables like gender, anal stenosis and recurrences were presented as frequencies and proportions while quantitative variables like age were presented as mean and standard deviation.

Table 1: Showing demographic data, degree of piles and type of operation

\begin{tabular}{lcc}
\hline Patients & Number & Percentage \\
\hline Total & 300 & \\
Male & 235 & $62.60 \%$ \\
Female & 65 & $37.40 \%$ \\
$2^{\text {nd }}$ degree & 52 & $17.30 \%$ \\
$3^{\text {rd }}$ degree & 161 & $53.70 \%$ \\
$4^{\text {th }}$ degree & & $29.00 \%$ \\
Primary & 87 & $88.10 \%$ \\
Redo & 264 & $11.90 \%$ \\
Milligan Morgan & 36 & $7.20 \%$ \\
Rubber band ligation & 22 & $4.00 \%$ \\
Stapled hemorroidectomy & 12 & $0.60 \%$ \\
\hline & 2 & Range \\
\hline Patients & & $18-72$ \\
\hline Age (year) & Mean & $15-30$ \\
Operative time (minute) & 34 & $1-4$ \\
Hospital stay (day) & 23 & $6-24$ \\
Follow up (month) & 1 &
\end{tabular}




\begin{tabular}{|c|c|c|}
\hline Complication & Number & Percentage \\
\hline \multicolumn{3}{|l|}{ Bleeding } \\
\hline No bleeding & 140 & $49.9 \%$ \\
\hline minor or less than 1 week & 92 & $30.7 \%$ \\
\hline Minor bleeding more than 1 week but less than 1 month. & 66 & $22.10 \%$ \\
\hline $\begin{array}{l}\text { - Persistent brisk bleeding requiring re-operation (treated by } \\
\text { stitches) }\end{array}$ & 1 & $0.30 \%$ \\
\hline \multicolumn{3}{|l|}{ Pain } \\
\hline absent or mild (VAS $<3$ ) first 24 hours & 83 & $27.80 \%$ \\
\hline mild to moderate pain (VAS 3-6) or pain less than one week & 175 & $58.20 \%$ \\
\hline $\begin{array}{l}\text { Severe (VAS >6) or more than one month but less than } 3 \\
\text { months. }\end{array}$ & 28 & $9.40 \%$ \\
\hline unexplained or persistent pain more than 3 months & 14 & $4.60 \%$ \\
\hline \multicolumn{3}{|l|}{ Other complications } \\
\hline $\begin{array}{l}\text { low perianal fistula at site of the silk suture fixation of the } \\
\text { dilator }\end{array}$ & 2 & $0.80 \%$ \\
\hline Flatus incontinence (redo after Milligan Morgan operation) & 1 & $0.20 \%$ \\
\hline Persistent of asymptomatic & 36 & $12.00 \%$ \\
\hline $\begin{array}{l}\text { Thrombosed external piles (drained in outpatients clinic under } \\
\text { local anesthesia) }\end{array}$ & 13 & $4.30 \%$ \\
\hline Anal fissure & 6 & $1.90 \%$ \\
\hline Urgency and tenesmus & 17 & $5.70 \%$ \\
\hline
\end{tabular}

\section{Tricks}

Our results were encouraging, though not faultproof, as shown by absence of major complications on the one hand and less operative time, postoperative pain and bleeding on the other hand. This outcome was due to standardization of the technique regardless of the surgeon and addition of these tricks:

- Good strapping of the gluteal fold to make the anus flat lying at the same level with peri-anal region. This will allow the anal dilator to be well fitted into the anal canal and together with the redesigned additional openings we included in the dilator, the edge of the dilator is high enough in the anal canal which prevent taking too low purse-string. This step is very important in case of cone-shaped anus. Too distally placed staple line could result in resection of anal squamous epithelium with persistent postoperative pain and greater likelihood of sphincter injuries. Low purse-string suture could result also in cutting through the hemorrhoidal cushions leading to affection of the continent state postoperatively.

- The purse-string should not be less than one $\mathrm{cm}$ above the edge of the dilator (one $\mathrm{cm}$ above dentate line). Mainly sub-mucosal bites should be taken and without in situ rotation of the anoscope to avoid any gaps which will eventually result in obtaining incomplete doughnut. Too high purse-string should also be avoided.

- The purse-string bites should mainly be submucosal and not over mucosa. Tightening of the sub-mucosal purse-string will invert and collect the mucosa and submucosa into the stapler house without any gaps. Tightening of over-mucosa purse string will evert parts of these tissues away from the stapler house with gaps formation which will lead to incomplete doughnuts. (Figure 10). 

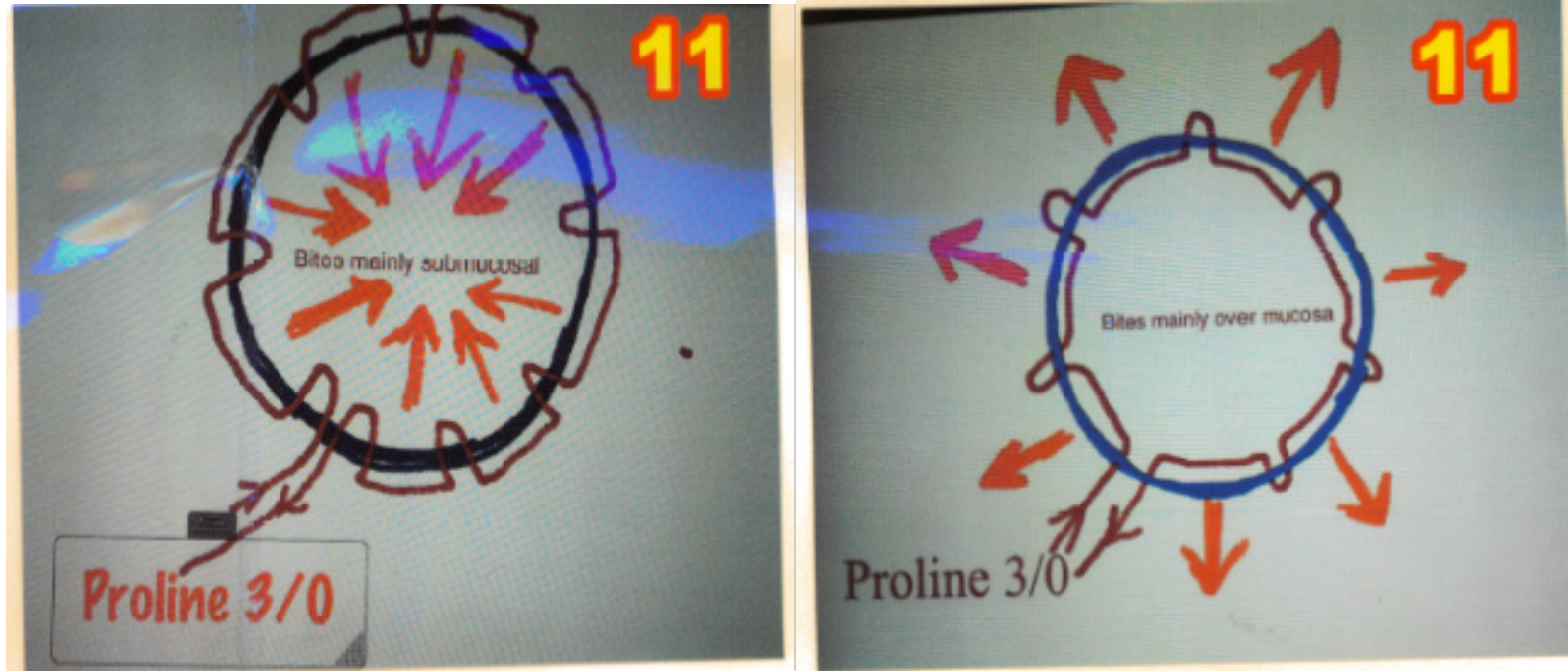

Fig 10: Sites of oversewing with proline.

- The purse-string shouldn't be too deep while doing it in redo cases and in the anterior rectal wall in female. Incorporation of the posterior vaginal wall or the rectal muscle has very bad sequelae and could result in recto-vaginal fistula, incontinence or septic complications. We check the posterior vaginal wall after the pursestring and after closure of the stapler.

- In case of huge prolapsed piles we don't use the purse string suture anoscope because the field will be totally obscured by the prolapsed mucosa. We take directly the purse-string over this prolapsed mucosa (Figure 11).

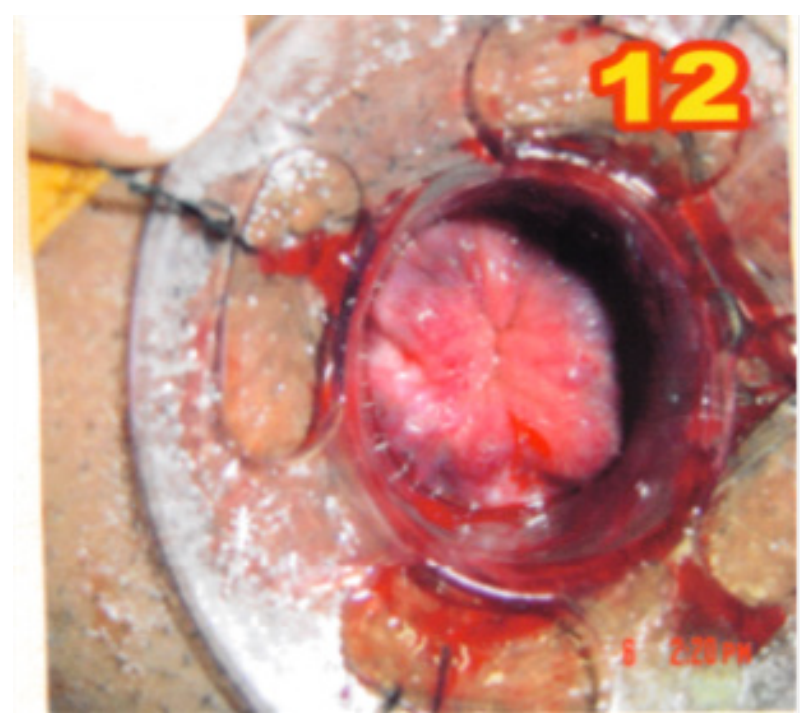

Fig 11.

We verify the integrity of the doughnut and the staple line at the end of the procedure by applying the purse string suture anoscope and taking reinforcement or hemostatic stitches in some patients. We try as much as possible to avoid these stitches except if highly indicated because it is one of the main causes of postoperative pain.

\section{Discussion}

Hemorrhoids are one of the commonly reported anorectal disorders and a worldwide problem. It has prevalence rate of $4.4 \%$ in the United States. ${ }^{19,20}$ Patients usually neglect the disease and hesitate to seek treatment. This leads to progression of the disease to grade III and IV hemorrhoids which are common. The disease is also under reported and neglected by general physicians due to social traditions, taboos and fear associated with proctoscopy. Most of the patients who presented late to the hospitals, presented with advanced disease (Grade III, IV or complicated Grade II). The treatment options for hemorrhoids are conservative (medical), nonsurgical (office based) and surgical treatments. A surgical option would be considered in patients with grade III \& grade IV hemorrhoids and concomitant anorectal pathology. ${ }^{21}$

Early in this study the operative time was about 60 minutes. With improvement in the learning curve and standardization of technique we achieved a drastic cut down in the operative time to be in the range of 15-30 minutes (mean 23 minutes).

These results agreed with Porwal as well as Elsebae and Hassan (2017) who reported that the average length of operation time was around 25 minutes. (range 20-45 min). ${ }^{18,21}$

The hospital stay in our series was short with 1 day for 282 patients $(94.1 \%)$, and 2-4 days for 18 patients $(5.9 \%)$. This lead to quicker recovery and return to daily activity.

This is in accordance with other authors who found that stapled hemorrhoidopexy procedure also resulted in shorter hospital stay, early recovery, 
and time to resume normal activities and overall improvement of symptoms and satisfaction. . $^{3,17,18,21}$

They reported that almost all patients were discharged on second to fourth day of surgery with smooth recovery and no any major postoperative event.

The mean follow up period for our patients was 20 months.

During follow up visits patients were assessed for complications and asked to fill up a pretested semi structured questionnaire. The patients who couldn't come for follow up were assessed by phone. At the end of one year follow up visit, screening proctoscopy was performed to assess the recurrence or prolapse and overall satisfaction.

Fortunately, we didn't encounter major mishaps as Stricture, Sphincter injury, Anastomotic dehiscence, rectal perforation or Septic complications.

When it comes to postoperative pain using VAS, our study recorded VAS $<3$ in $27.8 \%, 58.2 \%$ had VAS $3-6$, and $14 \%$ with VAS $>6$ during the hospital stay and follow up visits as scheduled.

This could be attributed to the fact that SH operation preserves the richly innervated anal canal tissue and perianal skin intact. Analgesic were prescribed according to patients' self pain assessment. $73.3 \%$ of patients required two doses of parenteral analgesia (Ketolac $\AA$ ) on first postoperative day, which reduced to a single dose in $60 \%$ of patients on second postoperative day.

Similar results of less postoperative pain, less analgesic requirements, and less pain at bowel movement were emphasized by Shrestha and his colleagues (2014). ${ }^{17}$

However, and contrary to our study and previous ones, Elsebae and Hassan 2017 reported that the VAS score was $\leq 3$ in $80 \%$ and $93.3 \%$ of patients on 1 st and 2 nd postoperative days respectively. ${ }^{18}$

In general, we reinforced the staple line with some stitches in cases of bleeding staple line after gun firing.

Though no bleeding incident occurred in $49.9 \%$ of patients during follow up $30.7 \%$ and $22.1 \%$ reported minor bleeding on the $1^{\text {st }}$ and $2^{\text {nd }}$ postoperative week, respectively and necessitating no active management.

One patient sustained persistent brisk bleeding 24 hours postoperatively and required reoperation and bleeding was controlled with a stitch at the anastomotic line (the site of bleeding).

Elsebae and Hassan (2017) observed that bleeding from the staple line after removal of the hemostatic gauze occurred in $23.35 \%$ of their patients and they required only cauterization of the bleeding spots with re-application of the hemostatic gauze for further 5 min. $^{18}$

The same authors noticed that minor delayed postoperative staple-line bleeding per rectum happened in $(46.7 \%)$ of patients in the first 48 hours, which did not require any intervention. However, minor bleeding persisted during 1st postoperative month in (20\%) of their patients then waned gradually and finally disappeared altogether at 3 months of follow-up. ${ }^{18}$

It is evident that internal mucosal prolapse is the most commonly seen co-morbid condition in Grade III and IV hemorrhoids that progress to external prolapse. The SH helps in treating this internal mucosal prolapse as well as lifting the external prolapse in better way hence the supposedly low recurrence rate.

There is no consistency among different studies regarding the finding of recurrent prolapse. Thus Porwal (2017) reported re-prolapse only in $(0.23 \%)$ of patients, making the incidence less than conventional surgery. ${ }^{21}$

Paradoxically, other studies had shown that SH procedure carries higher incidences of recurrence compared to conventional procedures..$^{9-13}$

In the present study we operated by conventional closed hemorrhoidectomy on 12 patients out of 15 for symptomatic prolapse after 12 months of the index surgery. Three patients were scheduled for surgery but they did not show up. We attribute our recurrence to the advanced grade of hemorrhoids that we operated upon as most of our cases were grade III and IV.

\section{Conclusion}

Although it is not popular in Egypt due to its expensive cost, Stapled hemorrhoidopexy is a safe procedure, with less operative pain, less hospital stay, and no major complications. we focused on some tips and tricks as well as our modification to the anoscope by adding extra openings at new sites aiming to make it more easy, more safe and more efficient to obtain the best results.

\section{References}

1. Lohsiriwat V: Treatment of hemorrhoids: A coloproctologist's view. World J Gastroenterol. 2015; 21(31): 9245-9252. 
2. Ala S, Saeedi M, Eshghi F, Mirzabeygi P: Topical metronidazole can reduce pain after surgery and pain on defecation in postoperative hemorrhoidectomy. Dis Colon Rectum. 2008; 51: 235-238.

3. Khalid Javed Abid, Muhammad Gul, Muhammad Nawaz Amin, Muhammad Tahir Saleem, Sadaf Ishaque: Comparison between open and stapled haemorrhoidectomy in the treatment of $3^{\text {rd }}$ and $4^{\text {th }}$ degree haemorrhoids at surgical unit II Shalamar Hospital Lahore, P J M H S. 2015; 9(4): $1144-1147$.

4. Arslani N, Patrlj L, Rajković Z, Papeš D, Altarac $S: \quad A$ randomized clinical trial comparing Ligasure versus stapled hemorrhoidectomy. Surg Laparosc Endosc Percutan Tech. 2012; 22: 58-61.

5. Sing WK: Hemorrhoidectomy with LigaSure ${ }^{T M}$ small jaw and stapled hemorrhoidopexy with $\mathrm{EEA}^{\mathrm{TM}}$ hemorrhoid and prolapse stapler with DST $^{\mathrm{TM}}$, clinical experience: The Science Behind Positive Patient Outcome. 2015, P1-6.

6. Pattana-arun J, Wesarachawit W, Tantiphlachiva K, Atithansakul P, Sahakitrungruang C, Rojanasakul A: A comparison of early postoperative results between urgent closed hemorrhoidectomy for prolapsed thrombosed hemorrhoids and elective closed hemorrhoidectomy. J Med Assoc Thai. 2009; 92: 1610-1615.

7. Alonso-Coello $P$, Mills $E$, Heels-Ansdell $D$, LópezYarto M, Zhou Q, Johanson JF, Guyatt G: Fiber for the treatment of hemorrhoids complications: A systematic review and meta-analysis. $\mathbf{A m ~} \mathbf{J}$ Gastroenterol. 2006; 101: 181-188. [7(17)].

8. Misra MC: Drug treatment of haemorrhoids. Drugs. 2005; 65: 1481-1491. [7(18)].

9. Perera $N$, Liolitsa D, Iype S, Croxford A, Yassin M, Lang $\mathrm{P}$, Ukaegbu O, van Issum C: Phlebotonics for haemorrhoids. Cochrane Database Syst Rev. 2012; 8:CD004322. [7(19)].

10. Beattie GC, Wilson RG, Loudon MA: The contemporary management of haemorrhoids. Colorectal Dis. 2002; 4: 450-454. [7(21)].

11. Thejeswi, Chandra P, Laxman, Kumar Y, Ram HS: Comparison of surgical treatment of hemorrhoids - stapled versus open and closed hemorrhoidectomy. The Internet Journal of Surgery. 2012; 28 (2). ISSN 1528-8242.
12. Milligan ETC, Naunton Morgan C, Jones L, Officer R: Surgical anatomy of the anal canal, and the operative treatment of hemorrhoids. Lancet. 1937; 230: 1119-1124. [8(4)].

13. Ferguson JA, Heaton JR: Closed hemorrhoidectomy. Dis Colon Rectum. 1959; 2: 176-179. [8(5)].

14. jun yang, Pei-Jing Cui, Hua-Zhong Han, Da-Nian Tong: Meta-analysis of stapled hemorrhoidopexy vs LigaSure hemorrhoidectomy, World $J$ Gastroenterol. 2013; 19(29): 4799-4807.

15. Ko-Chao Lee a, Hong-Hwa Chen a, KuanChih Chung $b$, Wan-Hsiang Hua, ChiaLo Chang a, Shung-Eing Lin a, Kai-Lung Tsai a, Chien-Chang Lu: Meta-analysis of randomized controlled trials comparing outcomes for stapled hemorrhoidopexy versus LigaSure hemorrhoidectomy for symptomatic hemorrhoids in adults, International Journal of Surgery. 2013; 11: 914-918.

16. Nienhuijs $S$, de Hingh I: Conventional versus LigaSure hemorrhoidectomy for patients with symptomatic Hemorrhoids. Cochrane Database Syst Rev. 2009; CD006761. [7(28)].

17. Shrestha S, Pradhan G.B.N, Shrestha R, Poudel P, Bhattachan C.L: Stapled Haemorrhoidectomy in the operative treatment of grade III and IV haemorrhoids, Nepal Med Coll J. 2014; 16(1): 72-74.

18. Elsebae MM, Hassan AMA: Stapled hemorrhoidopexy in egyptian patients with liver cirrhosis: Initial single institution experience, Clinics in Surgery. 2017 volume 2 article. 1392: 1-5.

19. Lacerda-Filho A, Silva RG: Stapled hemorrhoidectomy: present status. Arq Gastroenterol. 2005; 42(3): 191-4. [1(1)].

20. Shao WJ, Li GC, Zhang ZH, Yang BL, Sun GD, Chen YQ: Systematic review and meta-analysis of randomized controlled trials comparing stapled haemorrhoidopexy with conventional haemorrhoidectomy, Br J Surg. 2017 [1(2)].

21. Porwal AD: Stapled Hemorrhoidopexy and Long Term Outcomes, A single center experience of 3130 cases at clinic, India. Gastroenterology \& Hepatology: Open Access. 2017; 8(2): 00271. DOI: $10.15406 /$ ghoa. 